ESAIM: PROCEEDINGS, April 2007, Vol.17, 67-79

Alain Piétrus \& Michel H. Geoffroy, Editors

\title{
A METHOD FOR DETECTING POLLUTION IN DISSIPATIVE SYSTEMS WITH INCOMPLETE DATA.
}

\author{
Y. Miloudi ${ }^{1}$, O. Nakoulima ${ }^{2}$ And A. Omrane ${ }^{3}$
}

\begin{abstract}
Modelling environmental problems leads to mathematical systems with missing data. For instance, weather problems have generally missing initial conditions. The paper is concerned with identifying pollution terms arising in the state equation of some dissipative system with incomplete initial condition.

To this aim the so-called sentinel method is used. Here, the problem of determining a sentinel is equivalent to a null-controllability problem for which Carleman inequalities are revisited.
\end{abstract}

Résumé. La modélisation des problèmes environmentaux conduit à des systèmes mathématiques à données manquantes. C'est le cas par exemple des problèmes de météorologie où l'on ne connaît jamais la donnée initiale. Nous sommes concernés dans cet article, par l'identification des termes de pollution présents dans l'équation d'état d'un système dissipatif à donnée initiale incomplète.

Dans ce but, la méthode des sentinelles de Lions est utilisée. Ici, le problème de détermination d'une sentinelle est équivalent à un problème de contrôlabilité à zéro pour lequel on donne des estimations de type Carleman.

\section{INTRODUCTION}

Let be $T>0$, and $\Omega$ an open subset of $\mathbf{R}^{\mathbf{d}}$ of smooth boundary $\partial \Omega$, and denote by $\left.Q=\Omega \times\right] 0, T$ [ the space-time cylinder. We are intersted with systems partially known; we consider here the state equation :

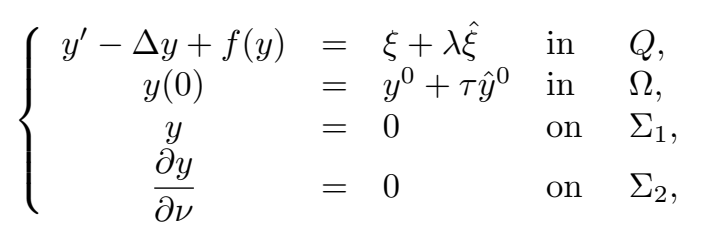

where $y=y(x, t ; \lambda, \tau)$, and where $\Sigma_{1}$ is a piece of the boundary $\left.\Sigma=\partial \Omega \times\right] 0, T\left[\right.$ and $\Sigma_{2}=\Sigma \backslash \Sigma_{1}$. We assume here that $f: \mathbf{R} \rightarrow \mathbf{R}$ is of class $C^{1}$, the functions $\xi$ and $y^{0}$ are known with $\xi \in L^{2}(Q)$ and $y^{0} \in L^{2}(\Omega)$. But, the terms : $\lambda \hat{\xi}$ (so-called pollution term) and $\tau \hat{y}^{0}$ (so-called perturbation term) are unknown, $\hat{\xi}$ and $\hat{y}^{0}$ are renormalized and represent the size of pollution and perturbation

$$
\|\hat{\xi}\|_{L^{2}(Q)} \leq 1, \quad\left\|\hat{y}^{0}\right\|_{L^{2}(\Omega)} \leq 1, \quad \text { so that the reals } \quad \lambda, \tau \quad \text { are small enough. }
$$

\footnotetext{
1 Département de Mathématiques, Université d'Oran Es-Sénia, BP No 1524 El M'Naouer, 31000 Oran (Algérie), email : miloudi@univ-oran.dz

2 Département de Mathématiques et Informatique, Université des Antilles et de La Guyane, Campus Fouillole 97159 Pointe à Pitre, Guadeloupe (FWI), email : onakouli@univ-ag.fr

3 email : aomrane@univ-ag.fr 
Some non empty open subset $O \subset \Omega$, is called observatory set. The observation is $y$ on $O$, for the time $T$. We denote by $y_{\text {obs }}$ this observation

$$
y_{o b s}=m_{o} \in L^{2}(O \times(0, T)) \text {. }
$$

We suppose that (1) has a unique solution denoted by $y(\lambda, \tau):=y(x, t ; \lambda, \tau)$ in some relevant space. The question is

how to calculate the pollution term $\lambda \hat{\xi}$ in the state equation, independently from the variation $\tau \hat{y}^{0}$ around the initial data?

Least squares. Question (3) is natural and leds to some developments; some answer is given by the least squares method. The method consists in considering the unknowns $\left\{\lambda \hat{\xi}, \tau \hat{y}^{0}\right\}=\{v, w\}$ as control variables, then the state $y(x, t ; v, w)$ has to be driven as close as possible to $m_{o}$.

This comes to some optimal control problem. By this way we look for the pair $(v, w)$, there is then no real possibility to find $v$ or $w$ independently.

Sentinels. The sentinel method of Lions [12] is a particular least squares method which is adapted to the identification of parameters in ecosystems with incomplete data; many models can be found in litterature. The sentinel concept relies on the following three objects : some state equation (for instance (1)), some observation function (2), and some control function $w$ to be determined.

Many papers use the definition of Lions in the theoretical aspect (see for example Bodart [1], Bodart-Fabre [3] $[4]$ ), as well as in the numerical one (see Bodart-Demeestere [2], Demeestere [6] and Kernevez [9]).

We now consider the sentinel method of Lions which is an other attempt and brings better answer to question (3), as we will explain now :

Let $h_{0}$ be some function in $L^{2}(O \times(0, T))$. Let on the other hand $\omega$ be some open and non empty subset of $\Omega$. For a control function $w \in L^{2}(\omega \times(0, T))$, we define the functional

$$
S(\lambda, \tau)=\int_{0}^{T} \int_{O} h_{0} y(\lambda, \tau) d x d t+\int_{0}^{T} \int_{\omega} \mathrm{w} y(\lambda, \tau) d x d t
$$

We say that $S$ defines a sentinel for the problem (1) if there exists w such that $S$ is insensitive (at first order) with respect the to missing terms $\tau \hat{y}^{0}$, which means

$$
\frac{\partial S}{\partial \tau}(0,0)=0
$$

for any $\hat{y}^{0}$ where here $(0,0)$ corresponds to $\lambda=\tau=0$, and if $\mathrm{w}$ minimizes the norm $\|\mathrm{v}\|_{L^{2}(\omega \times(0, T))}$.

Remark. The Lions sentinels $S$ assume $\omega=O$. In this case, the observation and the control share the same support, and the solution $\mathrm{w}=-h_{0}$ is trivial.

The definition (4) extends the one by Lions to the case where the observation and the control have different supports. This point of view (where $\omega \neq O$ ) has been considered for the first time by Nakoulima in [15]. Here we avoid the trivial solution.

Informations given by the sentinel. Because of (5) we can write

$$
S(\lambda, \tau) \simeq S(0,0)+\lambda \frac{\partial S}{\partial \lambda}(0,0), \quad \text { for } \quad \lambda, \tau \text { small }
$$


In (4), $S(\lambda, \tau)$ is observed and using (2),

$$
S(\lambda, \tau)=\int_{Q}\left(h_{0} \chi_{O}+\mathrm{w} \chi_{\omega}\right) m_{o} d x d t
$$

so that (5) becomes

$$
\lambda \frac{\partial S}{\partial \lambda}(0,0) \simeq \int_{Q}\left(h_{0} \chi_{O}+\mathrm{w} \chi_{\omega}\right)\left(m_{o}-y_{0}\right) d x d t
$$

with

$$
\frac{\partial S}{\partial \lambda}(0,0)=\int_{Q}\left(h_{0} \chi_{O}+\mathrm{w} \chi_{\omega}\right) y_{\lambda} d x d t,
$$

where here $\chi_{O}$ and $\chi_{\omega}$ denote the characteristic functions of $O$ and $\omega$ respectively.

The derivative $y_{\lambda}=(\partial y / \partial \lambda)(0,0)$ only depends on $\hat{\xi}$ and other known data. Consequently, the estimates (6) contains the informations on $\lambda \hat{\xi}$ (see for details remark 1.1 below).

\section{Equivalent CONTROLlability PROBlem}

We shall show in this section that the existence of a sentinel comes to null controllability property. We begin by transforming the insensibility condition (5).

Set

$$
y_{\tau}=\left.\frac{d}{d \tau} y(\lambda, \tau)\right|_{\lambda=\tau=0}
$$

Then the function $y_{\tau}$ is solution of

$$
\left\{\begin{array}{cllll}
y_{\tau}^{\prime}-\Delta y_{\tau}+f^{\prime}\left(y_{0}\right) y_{\tau} & = & 0 & \text { in } & Q \\
y_{\tau}(0) & = & \hat{y}^{0} & \text { in } & \Omega \\
y_{\tau} & = & 0 & \text { on } & \Sigma_{1} \\
\frac{\partial y_{\tau}}{\partial \nu} & = & 0 & \text { on } & \Sigma_{2}
\end{array}\right.
$$

where $y_{0}=y(0,0)$. Problem $(7)$ is linear and has a unique solution $y_{\tau}$ under mild assumptions on $f$.

The insensibility condition (5) holds if and only if

$$
\int_{Q}\left(h_{0} \chi_{O}+\mathrm{w} \chi_{\omega}\right) y_{\tau} d x d t=0
$$

We can transform (8) by introducing the classical adjoint state. More precisely, we define the function $q=q(x, t)$ as the solution of the backward problem :

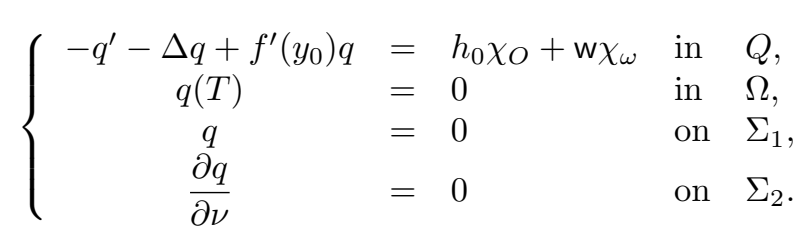

As for the problem (7), the problem (9) has a unique solution $q$ (under mild assumptions on $f^{\prime}\left(y_{0}\right)$ ). The function $q$ depends on the control w that we shall determine : 
Indeed, if we multiply the first equation in (9) by $y_{\tau}$, and we integrate by parts over $Q$, we obtain

$$
\int_{Q}\left(h_{0} \chi_{O}+\mathrm{w} \chi_{\omega}\right) y_{\tau} d x d t=\int_{\Omega} q(0) \hat{y}^{0} d x \quad \forall \hat{y}^{0}, \quad\left\|\hat{y}^{0}\right\|_{L^{2}(\Omega)} \leq 1 .
$$

So, the condition (5) (or (8)) is equivalent to

$$
q(0)=0
$$

This is a null-controllability problem.

Remark 1.1. The knowledge of the optimal control w provides informations about the pollution term $\lambda \hat{\xi}$. Indeed, denote by

$$
L=\frac{\partial}{\partial t}-\Delta+f^{\prime}\left(y_{0}\right) I_{d}
$$

and let $y_{\lambda}=\frac{\partial y}{\partial \lambda}(0,0)$ be the solution of

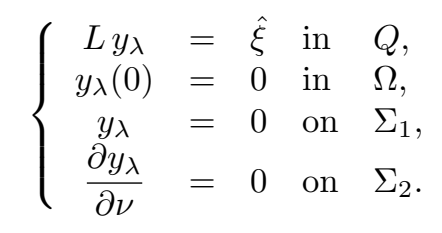

Integrating by parts, we then obtain

$$
\int_{Q} y_{\lambda} L^{*} q d x d t=\int_{Q} q \hat{\xi} d x d t
$$

with

So that from (9) and (6) we deduce

$$
L^{*}=-\frac{\partial}{\partial t}-\Delta+f^{\prime}\left(y_{0}\right) I_{d}
$$

$$
\int_{Q} \lambda \hat{\xi} d x d t=\int_{Q}\left(h_{0} \chi_{O}+\mathrm{w} \chi_{\omega}\right)\left(m_{o}-y_{0}\right) d x d t
$$

\section{Existence of A SEntinel}

We begin with some observability inequality, which will be proved in detail in the last section. Denote by

$$
\mathcal{V}=\left\{v \in \mathcal{C}^{\infty}(\bar{Q}) \text { such that : } v_{\left.\right|_{\Sigma_{1}}}=\left.\frac{\partial v}{\partial t}\right|_{\Sigma_{1}}=0 \text { and }\left.\frac{\partial v}{\partial \nu}\right|_{\Sigma_{2}}=0\right\}
$$

Then we have :

Theorem 2.1. Let be $u \in \mathcal{V}$, then there exists a positive constant $C=C\left(\Omega, \omega, O, T, f^{\prime}\left(y_{o}\right)\right)$ such that

$$
\int_{Q} \frac{1}{\theta^{2}}|u|^{2} d x d t \leq C\left[\int_{Q}|L u|^{2} d x d t+\int_{0}^{T} \int_{\omega}|u|^{2} d x d t\right]
$$

where $\theta \in \mathcal{C}^{2}(Q)$ positive with $\frac{1}{\theta}$ bounded. 
According to the RHS of (15), we consider the space $\mathcal{V}$ endowed with the bilinear form $a(.,$.$) defined by :$

$$
a(u, v)=\int_{Q} L u L v d x d t+\int_{0}^{T} \int_{\omega} u v d x d t .
$$

Let $V$ be the completion of $\mathcal{V}$ with respect to the norm

$$
v \mapsto\|v\|_{V}=\sqrt{a(v, v)}
$$

then, $V$ is a Hilbert space for the scalar product $a(v, \hat{v})$ and the associated norm.

Remark 2.2. We can precise the structure of the elements of $V$. Indeed, let $H_{\theta}(Q)$ be the weigthed Hilbert space defined by

$$
H_{\theta}(Q)=\left\{v \in L^{2}(Q) \quad \text { such that }: \quad \int_{Q} \frac{1}{\theta^{2}}|v|^{2} d x d t<\infty\right\},
$$

endowed with the natural norm $\|v\|_{\theta}=\left(\int_{Q} \frac{1}{\theta^{2}}|v|^{2} d x d t\right)^{\frac{1}{2}}$. This shows that $V$ is imbedded continuously $\|v\|_{\theta} \leq$ $C\|v\|_{V}$.

Now if $h_{0} \in L^{2}(Q)$ and $\theta h_{0} \in L^{2}(Q)$ (i.e. : $h_{0} \in L_{\theta}^{2}(Q)$ ), then from (15) and the Cauchy-Schwartz inequality, we deduce that the linear form defined on $V$ by

$$
v \mapsto \int_{Q} h_{0} \chi_{O} v d x d t
$$

is continuous. Therefore, from the Lax-Milgram theorem there exits a unique $u$ in $V$ solution of the variational equation :

$$
a(u, v)=\int_{Q} h_{0} \chi_{O} v d x d t \quad \forall v \in V
$$

Theorem 2.3. Assume that $h_{0} \in L_{\theta}^{2}(Q)$, and let $u$ be the unique solution of (18). We set

$$
\mathrm{w}=-u \chi_{\omega}
$$

and

$$
q=L u \text {. }
$$

Then, the pair $(\mathrm{w}, q)$ is such that (9)-(10) hold (i.e there is some insensitive sentinel defined by (4)-(5)).

\section{Proof of theorem 2.1}

The proof for the observability inequality in theorem 2.1 will hold from Carleman estimates that we carefully show in the following results.

Lemma 3.1. Let be $\omega_{0}$ an open set such that $\bar{\omega}_{0} \subset \omega$. Then there is $\psi \in C^{2}(\bar{\Omega})$ such that

$$
\left\{\begin{array}{cl}
\psi(x) & >0 \quad \forall x \in \Omega \\
\psi(x) & =0 \forall x \in \Gamma \\
|\nabla \psi(x)| & \neq 0 \quad \forall x \in \overline{\Omega-\omega_{0}}
\end{array}\right.
$$


Proof. See Imanuvilov [8]

We now use a function $\psi$ as given by the previous lemma, to define convenient weight functions. For $\lambda>0$, we set

$$
\varphi(x, t)=\frac{e^{\lambda \psi(x)}}{t(T-t)},
$$

and

$$
\eta(x, t)=\frac{e^{2 \lambda|\psi|_{\infty}}-e^{\lambda \psi(x)}}{t(T-t)} .
$$

Then

$$
\nabla \varphi=\lambda \varphi \nabla \psi, \quad \nabla \eta=-\lambda \varphi \nabla \psi
$$

We also notice the following properties :

$$
\begin{gathered}
\left|\frac{\partial \varphi}{\partial t}\right| \leq T \varphi^{2}, \quad\left|\frac{\partial^{2} \varphi}{\partial t^{2}}\right| \leq T^{2} \varphi^{3}, \\
\left|\frac{\partial \eta}{\partial t}\right| \leq T \varphi^{2}, \quad\left|\frac{\partial^{2} \eta}{\partial t^{2}}\right| \leq T^{2} \varphi^{3} .
\end{gathered}
$$

Remark 3.2. Note that $\eta$ increases to $+\infty$ when $t \rightarrow T$ or $t \rightarrow 0$, but $\eta$ is uniformly bounded on $\Omega \times[\delta, T-\delta]$ for any $\delta>0$.

On the other hand, for fixed $s>0$ the function $e^{-s \eta(x, t)}$ goes to 0 when $t \rightarrow T$ or $t \rightarrow 0$.

The following theorem states the Carleman inequalities concerning (14) :

Proposition 3.3. There exist constants $s_{0}>0, \lambda_{0}>0$ and $C>0$ depending on $\Omega, \omega$, $\psi$, and $T$, such that for all $s \geq s_{0}, \lambda \geq \lambda_{0}$, and for any function $u \in \mathcal{V}$ given by (14), we have

$$
\begin{array}{r}
2 s^{3} \lambda^{4} \int_{Q} \varphi^{3} e^{-2 s \eta}|u|^{2} d x d t+4 s^{2} \lambda \int_{\Sigma_{2}} \varphi \frac{\partial \eta}{\partial t} \frac{\partial \psi}{\partial \nu} e^{-2 s \eta}|u|^{2} d \gamma d t \\
-4 s^{3} \lambda^{3} \int_{\Sigma_{2}} \varphi^{3}|\nabla \psi|^{2} \frac{\partial \psi}{\partial \nu} e^{-2 s \eta}|u|^{2} d \gamma d t-4 s^{2} \lambda^{3} \int_{\Sigma_{2}}^{\varphi^{2}|\nabla \psi|^{2}} \frac{\partial \psi}{\partial \nu} e^{-2 s \eta}|u|^{2} d \gamma d t \\
-2 s \lambda \int_{\Sigma_{2}} \varphi \frac{\partial \psi}{\partial \nu} e^{-2 s \eta} \frac{\partial u}{\partial t} u d \gamma d t-4 s \lambda \int_{\Sigma_{1}} \varphi \nabla \psi e^{-2 s \eta} \nabla u \frac{\partial u}{\partial \nu} d \gamma d t \\
+2 s \lambda \int_{\Sigma_{\Sigma}} \varphi \frac{\partial \psi}{\partial \nu} e^{-2 s \eta}|\nabla u|^{2} d \gamma d t \\
\leq C\left(\int_{Q} e^{-2 s \eta}\left|\frac{\partial u}{\partial t}-\Delta u\right|^{2} d x d t+s^{3} \lambda^{4} \int_{0}^{T} \int_{\omega} \varphi^{3} e^{-2 s \eta}|u|^{2} d x d t\right) .
\end{array}
$$

Proof. We use the method by Fursikov and Imanuvilov [7], Imanuvilov [8], Lebeau and Robbiano [10], and Puel [16] [17] (case $\Sigma_{2}=\emptyset$ and $\Sigma_{1}=\Sigma$ ).

For $s \geq s_{0}$ and $\lambda \geq \lambda_{0}$, we define

$$
w(x, t)=e^{-s \eta(x, t)} u(x, t)
$$

We easily notice that

$$
w(x, 0)=w(x, T)=0 .
$$

Calculating $g=\left(\partial_{t}-\Delta\right)\left(e^{s \eta} w\right)$, with notation (27), we get

$$
P_{1} w+P_{2} w=g_{s}
$$


where

$$
\begin{aligned}
P_{1} w & =\frac{\partial w}{\partial t}+2 s \lambda \varphi \nabla \psi \nabla w+2 s \lambda^{2} \varphi|\nabla \psi|^{2} w \\
P_{2} w & =-\Delta w-s^{2} \lambda^{2} \varphi^{2}|\nabla \psi|^{2} w+s \frac{\partial \eta}{\partial t} w \\
g_{s} & =e^{-s \eta} g+s \lambda^{2} \varphi|\nabla \psi|^{2} w-s \lambda \varphi \Delta \psi w .
\end{aligned}
$$

Taking the $L^{2}$ norm we get :

$$
\int_{Q}\left|P_{1} w\right|^{2} d x d t+\int_{Q}\left|P_{2} w\right|^{2} d x d t+2 \int_{Q} P_{1} w P_{2} w d x d t=\int_{Q}\left|g_{s}\right|^{2} d x d t .
$$

We shall now calculate $\int_{Q} P_{1} w P_{2} w d x d t$. This will give 9 terms $I_{k, l}$.

In order to organize our calculus, we denote by $A$ and $B$ the quantities such that $A$ contains all the terms which can be upper bounded by

and by $B$ all those which can be bounded by

$$
c\left(s \lambda+\lambda^{2}\right) \int_{Q} \varphi|\nabla w|^{2} d x d t
$$

$$
c\left(s^{2} \lambda^{4}+s^{3} \lambda^{3}\right) \int_{Q} \varphi^{3}|w|^{2} d x d t .
$$

We denote by $\nu$ the outer normal on $\Gamma$. Note down that $\psi$ cancels on $\Gamma$. We then have the following results :

$$
\begin{aligned}
I_{1,1} & =-\int_{Q} \frac{\partial w}{\partial t} \Delta w d x d t \\
& =-\int_{\Sigma} \frac{\partial w}{\partial t} \frac{\partial w}{\partial \nu} d \gamma d t+0, \\
I_{1,2} & =-s^{2} \lambda^{2} \int_{Q} \frac{\partial w}{\partial t} \varphi^{2}|\nabla \psi|^{2} w d x d t=B, \\
I_{1,3} & =s \int_{Q} \frac{\partial w}{\partial t} \frac{\partial \eta}{\partial t} w d x d t=\frac{s}{2} \int_{Q} \frac{\partial \eta}{\partial t} \frac{\partial}{\partial t}\left(|w|^{2}\right) d x d t \\
& =-\frac{s}{2} \int_{Q} \frac{\partial^{2} \eta}{\partial t^{2}}|w|^{2} d x d t=B .
\end{aligned}
$$

And,

$$
\begin{aligned}
I_{2,1}= & -2 s \lambda \int_{Q} \varphi \nabla \psi \nabla w \Delta w d x d t \\
= & -2 s \lambda \int_{\Sigma} \varphi \nabla \psi \cdot \nabla w \frac{\partial w}{\partial \nu} d \gamma d t+2 s \lambda^{2} \int_{Q} \varphi|\nabla \psi \cdot \nabla w|^{2} d x d t \\
& +s \lambda \int_{\Sigma} \varphi \frac{\partial \psi}{\partial \nu}|\nabla w|^{2} d \gamma d t-s \lambda^{2} \int_{Q} \varphi|\nabla \psi|^{2}|\nabla w|^{2} d x d t+A,
\end{aligned}
$$




$$
\begin{aligned}
I_{2,2} & =-2 s^{3} \lambda^{3} \int_{Q} \varphi^{3}|\nabla \psi|^{2} \nabla \psi \nabla w \cdot w=-s^{3} \lambda^{3} \int_{Q} \varphi^{3}|\nabla \psi|^{2} \nabla \psi \nabla|w|^{2} \\
& =-s^{3} \lambda^{3} \int_{\Sigma} \varphi^{3}|\nabla \psi|^{2} \frac{\partial \psi}{\partial \nu}|w|^{2}+3 s^{3} \lambda^{4} \int_{Q} \varphi^{3}|\nabla \psi|^{4}|w|^{2}+B \\
I_{2,3} & =2 s^{2} \lambda \int_{Q} \varphi \nabla \psi \nabla w \frac{\partial \eta}{\partial t} w d x d t \\
& =s^{2} \lambda \int_{\Sigma} \varphi \frac{\partial \eta}{\partial t} \frac{\partial \psi}{\partial \nu}|w|^{2} d x d t+B .
\end{aligned}
$$

Finally :

$$
\begin{aligned}
I_{3,1} & =-2 s \lambda^{2} \int_{Q} \varphi|\nabla \psi|^{2} \Delta w \cdot w d x d t \\
& =-2 s \lambda^{2} \int_{\Sigma} \varphi|\nabla \psi|^{2} \frac{\partial w}{\partial \nu} \cdot w+2 s \lambda^{2} \int_{Q} \varphi|\nabla \psi|^{2}|\nabla w|^{2}+A+B \\
I_{3,2} & =-2 s^{3} \lambda^{4} \int_{Q} \varphi^{3}|\nabla \psi|^{4}|w|^{2} d x d t \\
I_{3,3} & =2 s^{2} \lambda^{2} \int_{Q} \varphi \frac{\partial \eta}{\partial t}|\nabla \psi|^{2}|w|^{2} d x d t=B
\end{aligned}
$$

Summing all the terms, it follows :

$$
\begin{aligned}
2 \int_{Q} P_{1} w P_{2} w d x d t=A & +B+2 s \lambda^{2} \int_{Q} \varphi|\nabla \psi|^{2}|\nabla w|^{2} d x d t \\
& +2 s^{3} \lambda^{4} \int_{Q} \varphi^{3}|\nabla \psi|^{4}|w|^{2} d x d t+4 s \lambda^{2} \int_{Q} \varphi|\nabla \psi \cdot \nabla w|^{2} d x d t \\
& -2 \int_{\Sigma} \frac{\partial w}{\partial t} \frac{\partial w}{\partial \nu} d \gamma d t-4 s \lambda \int_{\Sigma} \varphi \nabla \psi \cdot \nabla w \frac{\partial w}{\partial \nu} d \gamma d t \\
& +2 s \lambda \int_{\Sigma} \varphi \frac{\partial \psi}{\partial \nu}|\nabla w|^{2} d \gamma d t-2 s^{3} \lambda^{3} \int_{\Sigma} \varphi^{3}|\nabla \psi|^{2} \frac{\partial \psi}{\partial \nu}|w|^{2} d \gamma d t \\
& +2 s^{2} \lambda \int_{\Sigma} \varphi \frac{\partial \eta}{\partial t} \frac{\partial \psi}{\partial \nu}|w|^{2} d \gamma d t-4 s \lambda^{2} \int_{\Sigma} \varphi|\nabla \psi|^{2} \frac{\partial w}{\partial \nu} . w d \gamma d t
\end{aligned}
$$

But $|\nabla \psi| \neq 0$ on $\overline{\Omega-\omega_{0}}$, hence there is $\delta>0$ such that

$$
|\nabla \psi| \geq \delta \quad \text { on } \quad \overline{\Omega-\omega_{0}}
$$

On the other hand

$$
\int_{Q}\left|g_{s}\right|^{2} d x d t \leq \int_{Q} e^{-2 s \eta}|g|^{2} d x d t+B
$$


so that

$$
\begin{array}{r}
\int_{Q}\left|P_{1} w\right|^{2} d x d t+\int_{Q}\left|P_{2} w\right|^{2} \\
d x d t+2 \int_{Q} P_{1} w P_{2} w d x d t \\
\leq \int_{Q} e^{-2 s \eta}|g|^{2} d x d t+B
\end{array}
$$

Consequently :

$$
\begin{array}{r}
\int_{Q}\left|P_{1} w\right|^{2} d x d t+\int_{Q}\left|P_{2} w\right|^{2} d x d t+2 s \lambda^{2} \delta^{2} \int_{Q} \varphi|\nabla w|^{2} d x d t \\
+2 s^{3} \lambda^{4} \delta^{4} \int_{Q} \varphi^{3}|w|^{2} d x d t-2 \int_{\Sigma} \frac{\partial w}{\partial t} \frac{\partial w}{\partial \nu} d \gamma d t-4 s \lambda \int_{\Sigma} \varphi \nabla \psi \cdot \nabla w \frac{\partial w}{\partial \nu} d \gamma d t \\
+2 s \lambda \int_{\Sigma} \varphi \frac{\partial \psi}{\partial \nu}|\nabla w|^{2} d \gamma d t-2 s^{3} \lambda^{3} \int_{\Sigma} \varphi^{3}|\nabla \psi|^{2} \frac{\partial \psi}{\partial \nu}|w|^{2} d \gamma d t \\
+2 s^{2} \lambda \int_{\Sigma} \varphi \frac{\partial \eta}{\partial t} \frac{\partial \psi}{\partial \nu}|w|^{2} d \gamma d t-4 s \lambda^{2} \int_{\Sigma} \varphi|\nabla \psi|^{2} \frac{\partial w}{\partial \nu} \cdot w d \gamma d t+A+B \\
\leq \int_{Q} e^{-2 s \eta}|g|^{2} d x d t+B+2 s \lambda^{2} \delta^{2} \int_{0}^{T} \int_{\omega_{0}} \varphi|\nabla w|^{2} d x d t+2 s^{3} \lambda^{4} \delta^{4} \int_{0}^{T} \int_{\omega_{0}} \varphi^{3}|w|^{2} d x d t .
\end{array}
$$

We can eliminate $A$ and $B$ by choosing $s$ and $\lambda$ large enough. And we observe that :

$$
\begin{gathered}
\int_{0}^{T} \int_{\omega} \varphi \theta^{2}|\nabla w|^{2} d x d t \\
\leq C\left(\int_{Q} \varphi P_{2} w \theta^{2} w d x d t+\int_{0}^{T} \int_{\omega} \varphi^{\frac{1}{2}} \theta^{2}|\nabla w| \varphi^{\frac{1}{2}} w d x d t+s^{2} \lambda^{2} \int_{0}^{T} \int_{\omega} \varphi^{3} w^{2} d x d t\right),
\end{gathered}
$$

for $\theta \in \mathcal{D}(\omega)$ such that $0 \leq \theta \leq 1$ and $\theta(x)=1$ on $\omega_{0}$ gives

$$
2 s \lambda^{2} \delta^{2} \int_{0}^{T} \int_{\omega_{0}} \varphi|\nabla w|^{2} d x d t \leq \frac{1}{2} \int_{0}^{T} \int_{\Omega}\left|P_{2} w\right|^{2} d x d t+c s^{3} \lambda^{4} \int_{0}^{T} \int_{\omega} \varphi^{3}|w|^{2} d x d t
$$

Now, we should write the inequality below in terms of the solution $u$, since

$$
|w|^{2}=e^{-2 s \eta}|u|^{2}
$$


So,

$$
\begin{array}{r}
\frac{1}{2} \int_{Q}\left|P_{1} w\right|^{2} d x d t+\frac{1}{2} \int_{Q}\left|P_{2} w\right|^{2} d x d t \\
+2 s \lambda^{2} \int_{Q} \varphi|\nabla w|^{2} d x d t+2 s^{3} \lambda^{4} \int_{Q} \varphi^{3} e^{-2 s \eta}|u|^{2} d x d t \\
+2 s^{2} \lambda \int_{\Sigma} \varphi \frac{\partial \eta}{\partial t} \frac{\partial \psi}{\partial \nu} e^{-2 s \eta}|u|^{2} d \gamma d t-2 s^{3} \lambda^{3} \int_{\Sigma} \varphi^{3}|\nabla \psi|^{2} \frac{\partial \psi}{\partial \nu} e^{-2 s \eta}|u|^{2} d \gamma d t \\
-2 \int_{\Sigma} \frac{\partial w}{\partial t} \frac{\partial w}{\partial \nu} d \gamma d t-4 s \lambda \int_{\Sigma} \varphi \nabla \psi \cdot \nabla w \frac{\partial w}{\partial \nu} d \gamma d t \\
+2 s \lambda \int_{\Sigma} \varphi \frac{\partial \psi}{\partial \nu}|\nabla w|^{2} d \gamma d t-4 s \lambda^{2} \int_{\Sigma} \varphi|\nabla \psi|^{2} \frac{\partial w}{\partial \nu} \cdot w d \gamma d t \\
\leq C\left(\int_{Q} e^{-2 s \eta}|g|^{2} d x d t+s^{3} \lambda^{4} \int_{0}^{T} \int_{\omega} \varphi^{3} e^{-2 s \eta}|u|^{2} d x d t\right) .
\end{array}
$$

Now from

$$
\nabla u=e^{s \eta}(\nabla w-s \lambda \varphi \nabla \psi w)
$$

we deduce

$$
\int_{Q} \varphi e^{-2 s \eta}|\nabla u|^{2} d x d t \leq C\left(\int_{Q} \varphi|\nabla w|^{2} d x d t+s^{2} \lambda^{2} \int_{Q} \varphi^{3}|w|^{2} d x d t\right) .
$$

We then use the explicit form of $P_{1} w$ and $P_{2} w$, and get

$$
\frac{1}{s} \int_{Q} \frac{1}{\varphi}\left|\frac{\partial w}{\partial t}\right|^{2} d x d t \leq C\left(\int_{Q} e^{-2 s \eta}|g|^{2} d x d t+s^{3} \lambda^{4} \int_{0}^{T} \int_{\omega} \varphi^{3}|w|^{2} d x d t\right)
$$

and

$$
\frac{1}{s} \int_{Q} \frac{1}{\varphi}|\Delta w|^{2} d x d t \leq C\left(\int_{Q} e^{-2 s \eta}|g|^{2} d x d t+s^{3} \lambda^{4} \int_{0}^{T} \int_{\omega} \varphi^{3}|w|^{2} d x d t\right) .
$$

We sum up to finally have

$$
\begin{array}{r}
2 s^{3} \lambda^{4} \int_{Q} \varphi^{3} e^{-2 s \eta}|u|^{2} d x d t+4 s^{2} \lambda \int_{\Sigma} \varphi \frac{\partial \eta}{\partial t} \frac{\partial \psi}{\partial \nu} e^{-2 s \eta}|u|^{2} d \gamma d t \\
-4 s^{3} \lambda^{3} \int_{\Sigma} \varphi^{3}|\nabla \psi|^{2} \frac{\partial \psi}{\partial \nu} e^{-2 s \eta}|u|^{2} d \gamma d t-4 s^{2} \lambda^{3} \int_{\Sigma} \varphi^{2}|\nabla \psi|^{2} \frac{\partial \psi}{\partial \nu} e^{-2 s \eta}|u|^{2} d \gamma d t \\
+\int_{\Sigma}\left(2 s \frac{\partial \eta}{\partial t}-4 s \lambda^{2} \varphi|\nabla \psi|^{2}-4 s^{2} \lambda^{2} \varphi^{2}|\nabla \psi|^{2}\right) e^{-2 s \eta} \frac{\partial u}{\partial \nu} u \\
-2 s \lambda \int_{\Sigma}^{\varphi} \frac{\partial \psi}{\partial \nu} e^{-2 s \eta} \frac{\partial u}{\partial t} u d \gamma d t-4 s \lambda \int_{\Sigma} \varphi \nabla \psi e^{-2 s \eta} \nabla u \frac{\partial u}{\partial \nu} d \gamma d t \\
+2 s \lambda \int_{\Sigma} \varphi \frac{\partial \psi}{\partial \nu} e^{-2 s \eta}|\nabla u|^{2} d \gamma d t-2 \int_{\Sigma} e^{-2 s \eta} \frac{\partial u}{\partial t} \frac{\partial u}{\partial \nu} d \gamma d t \\
\leq C\left(\int_{Q} e^{-2 s \eta}|g|^{2} d x d t+s^{3} \lambda^{4} \int_{0}^{T} \int_{\omega} \varphi^{3} e^{-2 s \eta}|u|^{2} d x d t\right) .
\end{array}
$$

Using the fact that $u=\left.\frac{\partial u}{\partial t}\right|_{\Sigma_{1}}=0$, and $\left.\frac{\partial u}{\partial \nu}\right|_{\Sigma_{2}}=0$, we obtain (26). 
Now we proceed as the following. We define

$$
\widetilde{\varphi}(x, t)=\frac{e^{-\lambda \psi(x)}}{t(T-t)},
$$

and

Then

$$
\widetilde{\eta}(x, t)=\frac{e^{2 \lambda|\psi|_{\infty}}-e^{-\lambda \psi(x)}}{t(T-t)}
$$

$$
\nabla \widetilde{\varphi}=-\lambda \widetilde{\varphi} \nabla \psi, \quad \nabla \widetilde{\eta}=\lambda \widetilde{\varphi} \nabla \psi ;
$$

we still have the properties (24) and (25).

For $s \geq s_{0}$ and $\lambda \geq \lambda_{0}$, we define

$$
\widetilde{w}(x, t)=e^{-s \widetilde{\eta}(x, t)} u(x, t) .
$$

We easily notice that

$$
\widetilde{w}(x, 0)=\widetilde{w}(x, T)=0 .
$$

Calculating $\widetilde{P} \widetilde{w}=e^{-s \widetilde{\eta}} g=e^{-s \widetilde{\eta}}\left[\left(\partial_{t}-\Delta\right)\left(e^{s \widetilde{\eta}} \widetilde{w}\right)\right]$, using notation (48), we set

$$
\widetilde{P}_{1} \widetilde{w}+\widetilde{P}_{2} \widetilde{w}=\widetilde{g_{s}}
$$

where this time

$$
\begin{aligned}
\widetilde{P}_{1} \widetilde{w} & =\frac{\partial \widetilde{w}}{\partial t}-2 s \lambda \widetilde{\varphi} \nabla \psi \nabla \widetilde{w}+2 s \lambda^{2} \widetilde{\varphi}|\nabla \psi|^{2} \widetilde{w} \\
\widetilde{P}_{2} \widetilde{w} & =-\Delta \widetilde{w}-s^{2} \lambda^{2} \widetilde{\varphi}^{2}|\nabla \psi|^{2} \widetilde{w}+s \frac{\partial \widetilde{\eta}}{\partial t} \widetilde{w} \\
\widetilde{g_{s}} & =e^{-s \widetilde{\eta}} g+s \lambda^{2} \widetilde{\varphi}|\nabla \psi|^{2} \widetilde{w}+s \lambda \widetilde{\varphi} \Delta \psi \widetilde{w}
\end{aligned}
$$

We easily deduce the following result :

Proposition 3.4. There exists $s_{0}>0, \lambda_{0}>0$ and $C$ a positive constant depending on $\Omega, \omega, \psi$, and $T$, such that for all $s \geq s_{0}, \lambda \geq \lambda_{0}$, and for any fuction $u$ of (14), we have

$$
\begin{array}{r}
2 s^{3} \lambda^{4} \int_{Q} \widetilde{\varphi}^{3} e^{-2 s \widetilde{\eta}}|u|^{2} d x d t-4 s^{2} \lambda \int_{\Sigma_{2}} \widetilde{\varphi} \frac{\partial \widetilde{\eta}}{\partial t} \frac{\partial \psi}{\partial \nu} e^{-2 s \widetilde{\eta}}|u|^{2} d \gamma d t \\
+4 s^{3} \lambda^{3} \int_{\Sigma_{2}} \widetilde{\varphi}^{3}|\nabla \psi|^{2} \frac{\partial \psi}{\partial \nu} e^{-2 s \widetilde{\eta}}|u|^{2} d \gamma d t+4 s^{2} \lambda^{3} \int_{\Sigma_{2}} \widetilde{\varphi}^{2}|\nabla \psi|^{2} \frac{\partial \psi}{\partial \nu} e^{-2 s \widetilde{\eta}}|u|^{2} d \gamma d t \\
+2 s \lambda \int_{\Sigma_{2}} \widetilde{\varphi} \frac{\partial \psi}{\partial \nu} e^{-2 s \widetilde{\eta}} \frac{\partial u}{\partial t} u d \gamma d t+4 s \lambda \int_{\Sigma_{1}} \widetilde{\varphi} \nabla \psi e^{-2 s \widetilde{\eta}} \nabla u \frac{\partial u}{\partial \nu} d \gamma d t \\
-2 s \lambda \int_{\Sigma} \widetilde{\varphi} \frac{\partial \psi}{\partial \nu} e^{-2 s \widetilde{\eta}}|\nabla u|^{2} d \gamma d t \\
\leq C\left(\int_{Q} e^{-2 s \widetilde{\eta}}\left|\frac{\partial u}{\partial t}-\Delta u\right|^{2} d x d t+s^{3} \lambda^{4} \int_{0}^{T} \int_{\omega} \widetilde{\varphi}^{3} e^{-2 s \eta}|u|^{2} d x d t\right) .
\end{array}
$$

Proof. The proof is similar to the one of Proposition 3.3, so we let it to the reader.

Finally, we give below the conclusion to theorem 2.1. 
We obtain from Proposition 3.3 and Proposition 3.4 the following observability inequality :

Corollary 3.5. There is a positive constant $C=C(\Omega, \omega, \psi, T)$ such that we have

$$
\int_{Q} \frac{1}{\theta^{2}}|u|^{2} d x d t \leq C\left[\int_{Q}\left|\frac{\partial u}{\partial t}-\Delta u\right|^{2} d x d t+\int_{0}^{T} \int_{\omega}|u|^{2} d x d t\right]
$$

where $\frac{1}{\theta^{2}}=\varphi^{3} e^{-2 s \eta}+\widetilde{\varphi}^{3} e^{-2 s \tilde{\eta}}$ is a bounded weight function.

Proof. Summing the terms in (26) and (54) we get the following :

$$
\begin{gathered}
2 s^{3} \lambda^{4} \int_{Q}\left(\varphi^{3} e^{-2 s \eta}+\widetilde{\varphi}^{3} e^{-2 s \widetilde{\eta}}\right)|u|^{2} d x d t \\
-4 s^{2} \lambda \int_{\Sigma_{2}}\left(\varphi \frac{\partial \eta}{\partial t} e^{-2 s \eta}-\widetilde{\varphi} \frac{\partial \widetilde{\eta}}{\partial t} e^{-2 s \widetilde{\eta}}\right) \frac{\partial \psi}{\partial \nu}|u|^{2} d \gamma d t \\
-4 s^{3} \lambda^{3} \int_{\Sigma_{2}}\left(\varphi^{3} e^{-2 s \eta}-\widetilde{\varphi}^{3} e^{-2 s \widetilde{\eta}}\right)|\nabla \psi|^{2} \frac{\partial \psi}{\partial \nu}|u|^{2} d \gamma d t \\
-4 s^{2} \lambda^{3} \int_{\Sigma_{2}}\left(\varphi^{2} e^{-2 s \eta}-\widetilde{\varphi}^{2} e^{-2 s \widetilde{\eta}}\right)|\nabla \psi|^{2} \frac{\partial \psi}{\partial \nu}|u|^{2} d \gamma d t \\
+2 s \lambda \int_{\Sigma_{2}}\left(\varphi e^{-2 s \eta}-\widetilde{\varphi} e^{-2 s \widetilde{\eta}}\right) \frac{\partial \psi}{\partial \nu} \frac{\partial u}{\partial t} u d \gamma d t \\
-4 s \lambda \int_{\Sigma_{1}}\left(\varphi e^{-2 s \eta}-\widetilde{\varphi} e^{-2 s \widetilde{\eta}}\right) \nabla \psi \nabla u \frac{\partial u}{\partial \nu} d \gamma d t \\
+2 s \lambda \int_{\Sigma}\left(\varphi e^{-2 s \eta}-\widetilde{\varphi} e^{-2 s \widetilde{\eta}}\right) \frac{\partial \psi}{\partial \nu}|\nabla u|^{2} d \gamma d t \\
\leq C\left[\int_{Q}\left(e^{-2 s \eta}+e^{-2 s \widetilde{\eta}}\right)\left|\frac{\partial u}{\partial t}-\Delta u\right|^{2} d x d t\right. \\
\left.+s^{3} \lambda^{4} \int_{0}^{T} \int\left(\varphi_{\omega}^{3} e^{-2 s \eta}+\widetilde{\varphi}^{3} e^{-2 s \widetilde{\eta}}\right)|u|^{2} d x d t\right] .
\end{gathered}
$$

Now, it suffices to notice that $\varphi=\widetilde{\varphi}$ and $\eta=\widetilde{\eta}$ on $\Sigma$.

\section{REFERENCES}

[1] Bodart O., Application de la méthode des sentinelles à l'identification de sources de pollution dans un système distribué. Thèse de Doctorat, Université de Technologie de Compiègne, 1994.

[2] Bodart O., Demeestere P., Contrôle frontière de l'explosion en temps fini de la solution d'une équation de combustion. C.R. Acad. Sci., Série I, 325(8), 1997, pp $841-845$.

[3] Bodart O., Fabre C., Contrôle insensibilisant la norme de la solution d'une équation de la chaleur semi-linéaire. C.R. Acad. Sci., Série I, 316(8), 1993, pp $789-794$.

[4] Bodart O., Fabre C., Controls insensitizing the norm of a semilinear heat equation. Journ. of Math. Analysis and Applications, 195, 1995, pp $658-683$.

[5] Brezis H., Analyse fonctionnelle, Collection mathématiques appliquées pour la maîtrise, Masson, 1984.

[6] Demeestere P., Méthode des sentinelles : étude comparative et application à l'identification d'une frontière Contrôle du temps d'explosion d'une équation de combustion. Thèse de Doctorat, Université de Technologie de Compiègne, 1997.

[7] A. Fursikov, O. Yu. Imanuvilov, Controllability of evolution equations. Lecture Notes, Research Institute of Mathematics, Seoul National University, Korea (1996).

[8] O. Yu. Imanuvilov Controllability of parabolic equations. Sbornik Mathematics 186 : 6, 1995, pp $879-900$.

[9] Kernevez J.-P., The Sentinel Method and its Application to Environmental Pollution Problems, CRC. Press, Boca Raton, FL, 1997.

[10] Lebeau G., Robbiano L., Contrôle exacte de l'équation de la chaleur, Comm. P.D.E., 20, 1995, pp 335 - 356.

[11] Lions J.-L., Contrôle optimal de systèmes gouvernés par des équations aux dérivées partielles. Dunod, gauthier-Villars, Paris 1968. 
[12] Lions J.-L. Sentinelles pour les systèmes distribués à données incomplètes. Masson, Paris, 1992.

[13] Miloudi Y., Thèse d'Etat de l'Université d'Oran (to appear).

[14] Miloudi Y., Nakoulima O., Omrane A., Sentinels for dissipative systems of incomplete data (to appear).

[15] Nakoulima O., Contrôlabilité à zéro avec contraintes sur le contrôle. C. R. Acad. Sci. Paris, Ser. I 339,2004, pp 405 - 410.

[16] Puel J.-P., Contrôlablité approchée et contrôlabilité exacte. Notes de cours de D.E.A. Université Pierre et Marie Curie, Paris, 2001.

[17] Puel J.-P., Applications of global Carleman inequalities to controllability and inverse problems (to appear). 